\title{
High-throughput sequencing of microdissected chromosomal regions
}

\author{
Anja Weise ${ }^{1,10}$, Bernd Timmermann ${ }^{2,10}$, Manfred Grabherr ${ }^{3,10}$, Martin Werber ${ }^{4}$, Patricia Heyn ${ }^{5}$, \\ Nadezda Kosyakova ${ }^{1}$, Thomas Liehr ${ }^{1}$, Heidemarie Neitzel ${ }^{6}$, Kateryna Konrat ${ }^{6}$, Christiane Bommer ${ }^{5}$, \\ Carola Dietrich ${ }^{5}$, Anna Rajab ${ }^{7}$, Richard Reinhardt ${ }^{2}$, Stefan Mundlos ${ }^{5,8}$, Tom H Lindner ${ }^{9}$ and \\ Katrin Hoffmann ${ }^{\star, 5,8}$
}

The linkage of disease gene mapping with DNA sequencing is an essential strategy for defining the genetic basis of a disease. New massively parallel sequencing procedures will greatly facilitate this process, although enrichment for the target region before sequencing remains necessary. For this step, various DNA capture approaches have been described that rely on sequencedefined probe sets. To avoid making assumptions on the sequences present in the targeted region, we accessed specific cytogenetic regions in preparation for next-generation sequencing. We directly microdissected the target region in metaphase chromosomes, amplified it by degenerate oligonucleotide-primed PCR, and obtained sufficient material of high quality for highthroughput sequencing. Sequence reads could be obtained from as few as six chromosomal fragments. The power of cytogenetic enrichment followed by next-generation sequencing is that it does not depend on earlier knowledge of sequences in the region being studied. Accordingly, this method is uniquely suited for situations in which the sequence of a reference region of the genome is not available, including population-specific or tumor rearrangements, as well as previously unsequenced genomic regions such as centromeres.

European Journal of Human Genetics (2010) 18, 457-462; doi:10.1038/ejhg.2009.196; published online 4 November 2009

Keywords: genomic selection; enrichment; microdissection; next-generation sequencing

\section{INTRODUCTION}

Despite recent advances in sequencing technologies, present capabilities do not permit routine whole-genome sequencing for mutation detection. In response, enrichment methods have been described to capture specific sequences from genomes that will work well for most screening studies. ${ }^{1-7}$

However, these capture-based enrichment methods are limited in some situations; they require an earlier knowledge of the target sequences for array or primer design and are thus restricted to resequencing projects. They will not be suitable when rare sequence rearrangements are in place; for example, inter-individual differences of highly dynamic structures, such as telomere and subtelomere regions, that might be difficult to capture by the mentioned methods but have relevance to ageing, cancer and inherited disease. ${ }^{8-11}$ Other regions, such as pericentromeric heterochromatin, were not targeted by the Human Genome Project because they are difficult to clone and to annotate owing to high repeat content and homology. ${ }^{12}$ However, heterochromatin comprises $20 \%$ of the human genome, and seems to have relevance for gene expression and disease. ${ }^{13-15}$ In some linkage or association studies, significant results identify regions that contain no known genes. ${ }^{16,17}$ Furthermore, even regions with known genes could feature unrecognized rearrangements or insertion of mobile elements with effects on gene regulation and expression. ${ }^{18-20}$ Other frequent examples are cryptic rearrangements in promoter regions or fusion genes in cancer development. ${ }^{21,22}$ Those dynamics would be missed or difficult to analyze by the above-mentioned capture methods. Genome-wide paired-end sequencing is extremely sensitive, ${ }^{23}$ but may not be meaningful or practicable for large-scale screening studies when there already is a localized region of interest. However, nextgeneration sequencing technologies are developing extremely fast and will probably enable whole-genome sequencing at affordable costs in the near future.

To avoid making a priori assumptions on the sequences in the target region, we developed an approach that could directly start with a patient's chromosomal region linked to a disease. The most direct way is to dissect that suspicious piece of chromosome and sequence it. We have done that by coupling conventional cytogenetics (karyotyping), microdissection and high-throughput sequencing (Figure 1). We present data from three experiments, in which we obtained sequences from as few as six chromosomes and present a proof-of-feasibility protocol.

\footnotetext{
${ }^{1}$ Institute of Human Genetics and Anthropology, Jena, Germany; ${ }^{2}$ Max Planck Institute for Molecular Genetics, Service, Berlin, Germany; ${ }^{3}$ Broad Institute of Massachusetts Institute of Technology (MIT) and Harvard, Cambridge, MA, USA; ${ }^{4}$ Max Planck Institute for Molecular Genetics, Developmental Genetics, Berlin, Germany; ${ }^{5}$ Institute of Medical Genetics, Charité University Medicine, Berlin, Germany; ${ }^{6}$ nstitute for Human Genetics, Charité University Medicine, Berlin, Germany; ${ }^{7}$ Genetic Unit, Ministry of Health, Muscat, Sultanate of Oman; ${ }^{8}$ Department Development and Disease, Max Planck Institute for Molecular Genetics, Berlin, Germany; ${ }^{9}$ Division of Nephrology, Department of Medicine, Neurology, and Dermatology, University Clinic Leipzig, Leipzig, Germany

${ }^{*}$ Correspondence: Dr K Hoffmann, Institute of Medical Genetics, Charité University Medicine, Augustenburgerplatz 1, Berlin 13353, Germany.

Tel: +49 30450569 129/122; Fax: +49 30450569 915; E-mail: khoffma@gmx.de

10 These authors contributed equally to this work.

Received 30 September 2008; revised 11 August 2009; accepted 25 September 2009; published online 4 November 2009
} 

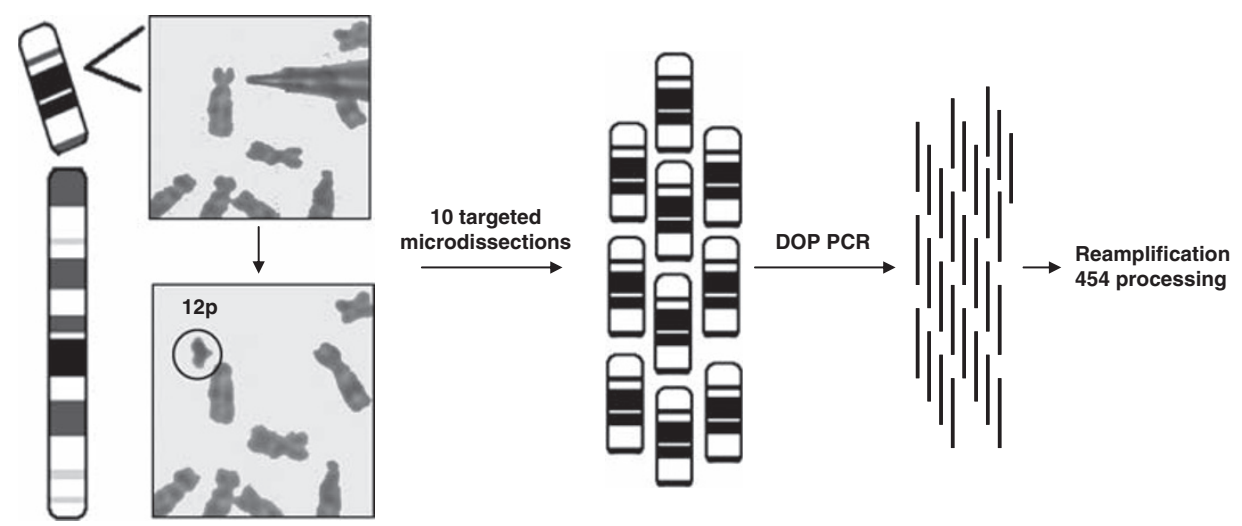

Figure 1 Microdissection-based enrichment for next-generation sequencing. The figure shows metaphase chromosomes prepared from lymphoblastoid cells and microdissection of chromosomal region 12p. In this experiment, we microdissected and processed 10 short arms of chromosome 12 . The microdissected fragments were amplified by DOP-PCR, followed by the processing protocol for 454 sequencing.
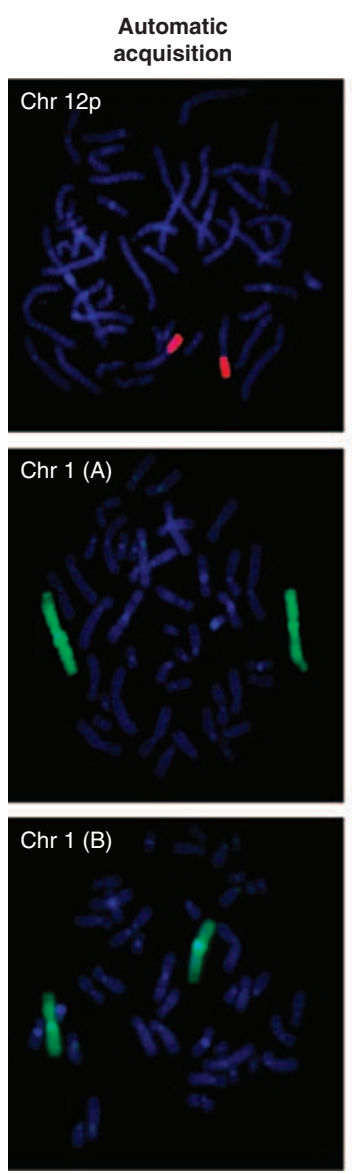
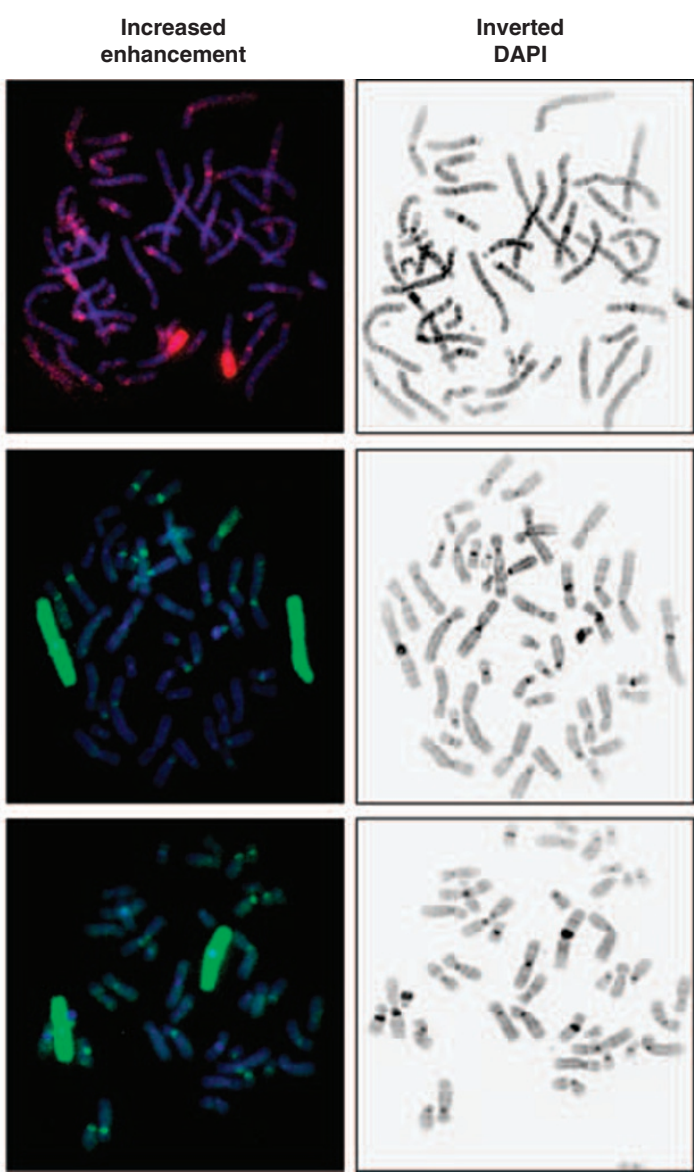

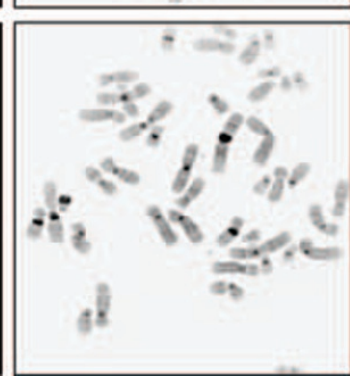

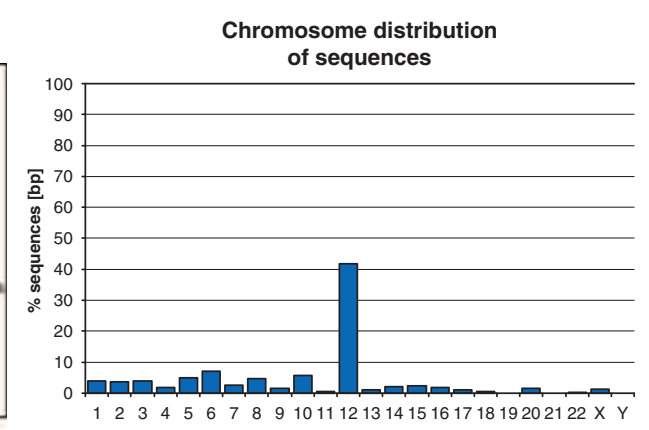
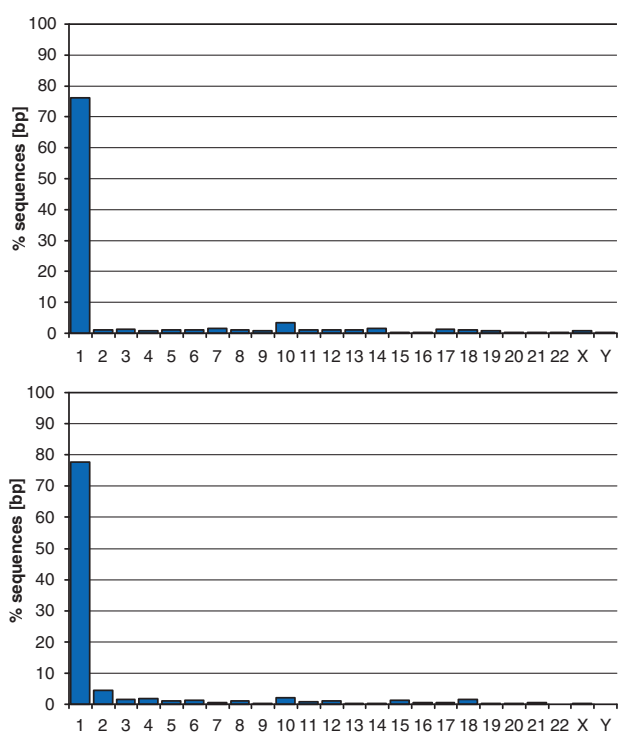

Figure 2 Specificity of the microdissected material tested in metaphase fluorescence in situ hybridization (FISH) and in 454 sequencing. Left panel: specificity of the microdissected material tested on metaphase FISH. On the left, the hybridization signals of the microdissected material are shown as seen under the microscope; material from chromosome $12 \mathrm{p}$ and whole chromosome 1 were purified and processed without major contamination. When signal detection was enhanced (middle panel), we saw minor cross-hybridization on other chromosomes, mainly in pericentromeric regions, which have a high degree of homology among chromosomes. The other faint cross-hybridizations might be due to repeat elements and segmental duplications. The inverted DAPI channel shows a GTG-like chromosome banding to unambiguously identify the metaphase chromosomes. Right panel: mappings of sequence reads show enrichment of the targeted chromosomes. A total of $41.8 \%$ of reads that uniquely mapped to the whole genome, aligned to targeted chromosome $12 \mathrm{p}$, and 76.2 and $77.6 \%$ to targeted chromosome 1 (experiments $A$ and $B$, respectively). This result documents the feasibility of the microdissection approach. Mapping on other chromosomes might represent repeat elements, transposons, gene families or annotation problems. The higher proportion of off-target hits from the microdissected material from chromosome $12 \mathrm{p}$ might result from the lower ratio of specific sequence to repeat-rich heterochromatin, as the microdissected short arm $12 p$ contains a significant amount of pericentromeric heterochromatin. In addition, $12 p$ is known to have been involved in segmental duplications. ${ }^{30-33}$ Also, annotation errors due to unclonable genomic regions with subsequent contig gaps might contribute. ${ }^{29}$ (For each experiments we used $1 / 16$ of a 454/Roche FLX run). 


\section{METHODS}

Preparation of metaphase chromosomes, microdissection and degenerate oligonucleotide-primed PCR

We prepared metaphase chromosomes from lymphoblastoid cells (chromosome 12p) and from peripheral blood (chromosome 1). Microdissection was performed as described. ${ }^{24}$ For amplification, we used an adapted degenerate oligonucleotide-primed PCR (DOP-PCR). ${ }^{24,25}$ A detailed protocol for microdissection and DOP-PCR is provided in the supplementary material. Before preparing the 454 library, we verified the specificity of the microdissected material by dye-labeling an aliquot of DOP-PCR product and subsequent hybridization on metaphase chromosomes (reverse painting, reverse fluorescence in situ hybridization, FISH). ${ }^{26}$

\section{Library preparation}

The 454 library was prepared according to the manufacturer's instruction, and included adapter ligation, library immobilization, melting and quantification. We performed an additional reamplification of the 454 library to get a measurable amount of library material. For this purpose, we used the normal Roche 454 amplification primer ( $20 \mu \mathrm{m}$ final concentration; Roche, Branford, CT, USA) and performed a standard PCR with 35 cycles ( $50 \mu$ l volume). On the basis of the short length of DOP-PCR products $(<200 \mathrm{bp})$ in the starting material for library preparation, we have not used paired-end sequencing.

\section{Sequencing runs carried out with 454/Roche FLX genome sequencer}

Runs were carried out according to the manufacturer's instructions with the following modification. To increase the number of sequencing reads, we passed the normal AMPure Bead Purification for FLX runs, Agencourt Bioscience Corporation, Beverly, MA, USA. We used $70 \times 75$ PTPs with 16-region gaskets. To obtain more sequencing information, we loaded the single lanes with more than 70000 DNA beads. Each experiment was processed in a 1/16 run.

\section{Bioinformatic analyses}

We mapped sequences against the genomic reference sequence (hg18, March 2006, build 36.1) using MegaBlast, National Center for Biotechnology Information, http://www.ncbi.nlm.nih.gov/blast/megablast.shtml. Although usually the aim is to maximize the amount of mapped reads, in our analysis, we put strong emphasis on a stringent discrimination between on- and off-target hits.
We determined an optimal $e$-value threshold that maximizes the number of unique hits, as described in Albert et al. ${ }^{2}$ Reads with multiple hits and significant $e$-values were considered as non-unique mappings and were excluded from further analysis. Thus, the amount of not mapped reads is the direct consequence of the stringent regime of parameters, enabling optimal ontarget off-target discrimination, and not because of other reasons such as contaminations, gaps or low sequencing quality.

\section{RESULTS}

For microdissection, we prepared metaphase chromosomes from human peripheral blood and from lymphoblastoid cells. We targeted human chromosomes $12 \mathrm{p}$ and 1 . We microdissected 10 short arms from chromosome 12 (experiment $\mathrm{Chr} 12 \mathrm{p}$ ) and 6 chromosomes 1 . The experiment for chromosome 1 was performed twice (experiments $\mathrm{Chr} 1(\mathrm{~A})$ and $\mathrm{Chr}$ 1(B), respectively). The critical step was amplification from small amounts of starting material, which was successfully done by DOP-PCR. We obtained sufficient amount of DOP-PCR product. Subsequently, we took an aliquot of microdissected and DOP-PCR-amplified material for proof of specificity on control chromosomes (reverse FISH in the left panel of Figure 2).

The remaining material was used for the 454 library preparation. Of all obtained sequence reads, about 52, 67 and 55\% could be mapped to the human reference genome in experiments $\operatorname{chr} 12 \mathrm{p}, \operatorname{chr} 1(\mathrm{~A})$ and chr1(B), respectively (Table 1). For chromosome $12 p, \sim 42 \%$ of sequence reads, that were mapped to the whole genome just once, had their primary BLAST hit in the target region (Table 1, Figure 2 upper right panel, Figure 3, Supplementary Figure 1). In both chromosome 1 experiments, more than $75 \%$ of uniquely mapped sequence reads had their hit on chromosome 1 (Table 1, Figure 2 middle and lower right panels, Figure 3, Supplementary Figure 1).

The distribution of sequencing coverage (Table 2, Figure 3, Supplementary Figure 1), the number of reads partially containing repeat sequences (Table 3, Figure 3, Supplementary Figure 1) and SNP detection rates (Table 1) were within the expected range for currently available enrichment methods. Although the sequence harvest can be

Table 1 Analysis of mapped and unmapped sequences regarding the target chromosome and sequence

\begin{tabular}{|c|c|c|c|}
\hline Parameter & Chr $12 p$ & $\operatorname{Chr} 1(A)$ & Chr 1(B) \\
\hline Obtained sequences (reads; $n$ ) & 998 & 1274 & 1416 \\
\hline Unmapped reads $(n)$ & 480 & 423 & 634 \\
\hline Mapped reads $(n)$ & 518 & 851 & 782 \\
\hline Percentage of mapped sequences (\%) & 51.9 & 66.8 & 55.2 \\
\hline Reads with unique genomic mapping & 368 & 589 & 550 \\
\hline Mapped sequences on target $\mathrm{CHR}(n)$ & 154 & 449 & 427 \\
\hline Percentage of uniquely mapped sequences on target (\%) & 41.8 & 76.2 & 77.6 \\
\hline Mapped read sizes on target $\mathrm{CHR}(\mathrm{bp})$ & 28349 & 73275 & 71993 \\
\hline Mapped read matches (bp) & 28243 & 72856 & 71579 \\
\hline $\operatorname{SNPS}(n)$ & 106 & 419 & 414 \\
\hline SNPs every how many base pairs & 267.44 & 174.88 & 173.90 \\
\hline Mapped sequences outside target $(n)$ & 214 & 140 & 123 \\
\hline Mapped read sizes outside target CHR (bp) & 37602 & 21399 & 21230 \\
\hline Mapped read matches (bp) & 37481 & 21285 & 21156 \\
\hline SNPS $(n)$ & 121 & 114 & 74 \\
\hline Average spacing of detected SNPs (bp) & 310.76 & 187.71 & 286.89 \\
\hline
\end{tabular}

It is to be noted that mapped reads contain all mapped reads, that is, unique chromosomal hits and sequences that hit twice or multiple times at different chromosomal positions. (For example, among 851 total-mapped reads in experiment Chr 1(A), 589 reads hit once throughout the genome. Off those 589 sequences, 449 mapped to the target chromosome and 140 outside the target). 


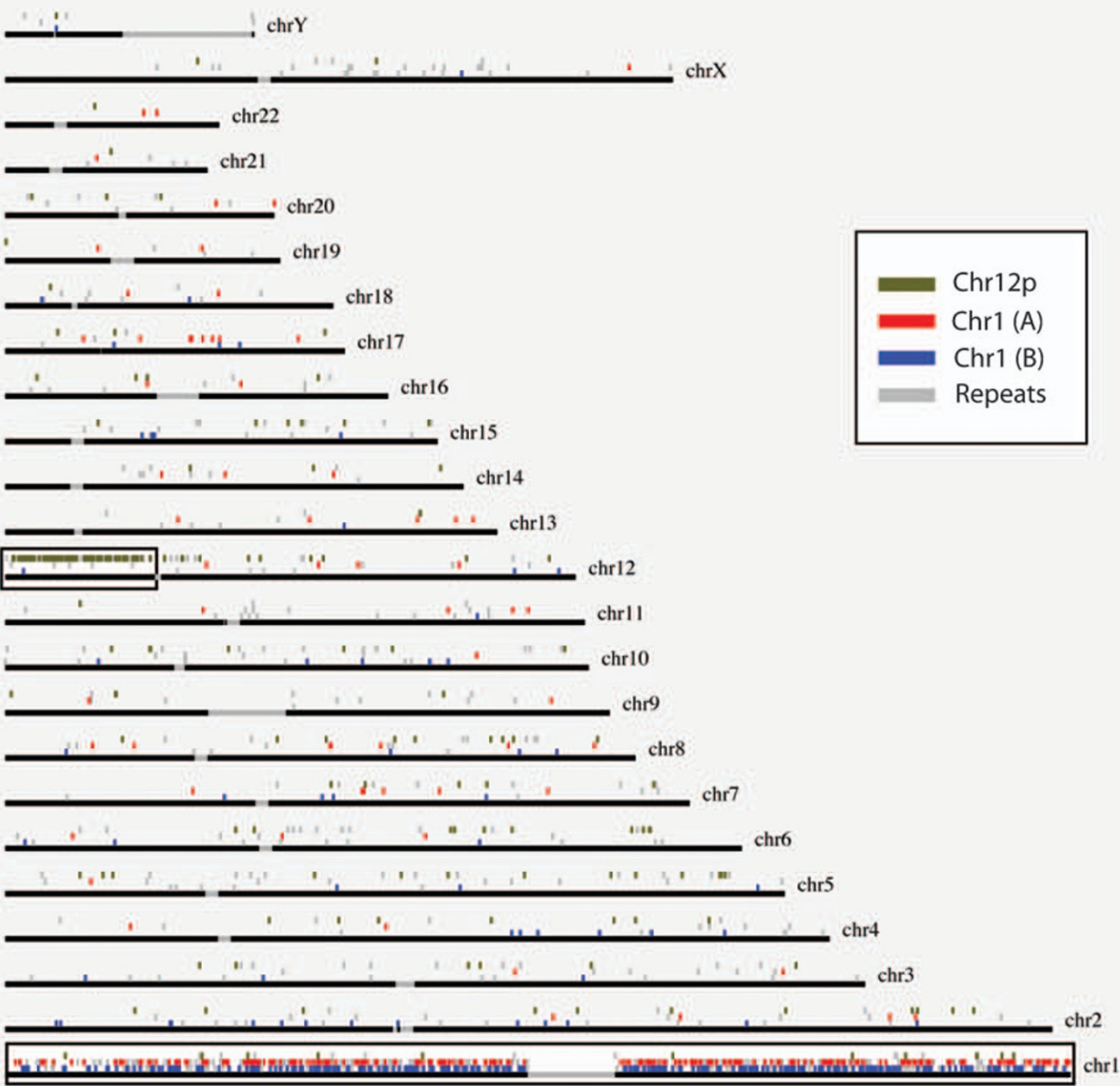

Figure 3 Distribution of reads mapped to the genome. For each chromosome, we show the unique alignment locations of reads from the three data sets $12 \mathrm{p}$, $1(\mathrm{~A})$ and 1 (B) (in olive, red and blue), as well as placements in annotated repeats (gray). The targeted regions (chromosome 1 and the $p$ arm of chromosome 12) are highlighted in white boxes (for a zoom-in of these regions, see Supplemental Figure 1).

Table 2 Distribution of multiple sequence coverage (analysis of read clusters)

\begin{tabular}{lrrr}
\hline Fold coverage & Chr 12p & Chr 1 (A) & Chr 1(B) \\
\hline 1 & 352 & 579 & 571 \\
2 & 50 & 76 & 59 \\
3 & 6 & 20 & 15 \\
4 & 1 & 7 & 6 \\
5 & 0 & 2 & 0 \\
6 & 1 & 2 & 0 \\
7 & 0 & 0 & 1 \\
8 & 1 & 0 & 1 \\
9 & 0 & 0 & 1 \\
10 & 1 & 1 & 0 \\
20 & 1 & 0 & 0 \\
\hline
\end{tabular}

In all three experiments, the majority of hit regions was covered only by one read. further optimized, we obtained a sufficient number of high-quality reads for a proof of principle. The data show that 454 sequencing, starting from as few as six chromosomes, is feasible.

\section{DISCUSSION}

We present the feasibility of a cytogenetic-based approach to capture target regions for next-generation sequencing. Direct microdissection of the target region in metaphase chromosomes with subsequent DOP-PCR amplification obtained sufficient material and quality for 454 sequencing from as few as six chromosomes.

We analyzed whether some fragments were sequenced several times by clustering reads. In all three experiments, the majority of hit regions were covered by only one read, indicating a coverage distribution and range for preferential amplification within the expected range. Although the representation of repeat regions in the obtained reads is higher than the average density in the currently available genome 
Table 3 Analysis of sequence repeat patterns

\begin{tabular}{|c|c|c|c|c|c|c|}
\hline & \multicolumn{2}{|c|}{ Exp. Chr $12 p$} & \multicolumn{2}{|c|}{ Exp. $\operatorname{Chr} 1(A)$} & \multicolumn{2}{|c|}{ Exp. Chr 1(B) } \\
\hline & On target & Off target & On target & Off target & On target & Off target \\
\hline \multicolumn{7}{|l|}{ Sequence reads with } \\
\hline LINE $(n)$ & 48 & 10 & 158 & 41 & 145 & 38 \\
\hline $\operatorname{LTR}(n)$ & 29 & 66 & 50 & 12 & 34 & 23 \\
\hline Simple repeat $(n)$ & 2 & 21 & 9 & 1 & 0 & 0 \\
\hline Reads without repeats $(n)$ & 72 & 31 & 182 & 53 & 192 & 47 \\
\hline Total reads $(n)$ & 154 & 214 & 449 & 140 & 427 & 123 \\
\hline Total sequence (bp) & 28349 & 37602 & 73275 & 21399 & 71993 & 21230 \\
\hline Density of repeats (bp) & 298.41 & 205.48 & 274.44 & 245.97 & 306.35 & 279.34 \\
\hline
\end{tabular}

SINE, small interspersed repetitive element (such as Alu repeats); LINE, large interspersed repetitive element (such as L1 elements); LTR, Iong terminal repeat.

The table shows the reads containing repetitive sequences vs those without repeats. On average, we detected one repeat every $300 \mathrm{bp}$ (last line of the table). According to a database analysis, for example, in chromosome 1, there is one repeat every $600 \mathrm{bp}$ on average. Thus, the prevalence of repetitive sequences is slightly overrepresented in our experiments. That might be due to incomplete repeat annotations in the current version of the human genome build as well as to new, not yet annotated private repetitive sequences.

annotation, the obtained data seem consistent in light of the many newly detected, probably population-specific or private insertions of repeat elements, as they become available from the Watson and Venter genome or the 1000 Genomes Project, respectively. ${ }^{23,27}$ Although it was not the aim of our experiments, analyzing regions with repeat elements might be facilitated by a microdissection approach. However, considering the fact that even more stable regions of the genome, such as exons, require a high sequencing depth, ${ }^{28}$ exploiting sequence variations in repeat elements will probably warrant an even higher coverage. Their de novo annotation would be facilitated by longer sequencing reads to include sequences adjacent to the repeat.

Making chromosomes visible requires the chromatin to condense and arrange, which happens mainly when cells replicate and prepare to divide (metaphase in cell cycle). Accordingly, direct microdissection of the patient's chromosomes is possible only when dividing cells are available. This state-of-affairs is a limitation. Another point to care for is the risk of contamination, especially during the microdissection process and the first cycles of DOP-PCR. However, single-cell techniques are well established in many pre-implantation diagnostic and tumor microdissection laboratories. The number of generated sequences is probably lower than that in other approaches, but can be increased by optimized loading density and compensated by more runs. Our approach works well for complete chromosomes and partial chromosome arms and regions. Smaller parts can also be microdissected, as was previously shown for microdissection libraries with band-resolutions that were created for chromosome painting. ${ }^{24}$ Such adaptation might be useful for specific questions. Here, we wanted to cover complete regions, including centromeric regions and repeats. When not required, repeats can be blocked by COT-1 DNA to increase the harvest for unique sequences.

We showed that high-throughput sequencing of microdissected chromosomes is feasible and can be done from a few molecules. The coupling of microdissection and next-generation sequencing is suited for a wide range of applications, including standard mutation detection. Sequencing phase-defined chromosomes allows experimental determination of haplotypes and haplotype blocks. The combination of defined localization information and independency from earlier knowledge of sequence composition in the target region might help in solving annotation problems of repeat rich or nonclonable regions in de novo sequencing. ${ }^{29}$ The approach might also be relevant in humans, when population-specific insertions are suspected, for tracking down small 'private' cytogenetic abnormalities in patients or tumor cells and for resequencing of dynamic chromosomal regions, such as telomeres, subtelomeres or pericentromeric heterochromatin.

\section{CONFLICT OF INTEREST}

The authors declare no conflict of interest.

\section{ACKNOWLEDGEMENTS}

We thank Anna Kosiura, Isabelle Kühndahl, Christina Roehr and Phillipe Schroeter for their technical expertise. We thank Mathias Meyer for his advice. Friedrich C Luft and Eddy Rubin critically read the manuscript. The Deutsche Forschungsgemeinschaft (DFG SFB 577 project A4 to KH, LI820/11-1 and LI820/17-1 to TL, Li768/6-1 and 6-2 to THL), the IZKF Jena (Start-up S16), the Stiftung Leukämie and the University Jena supported the study. Katrin Hoffmann received a Rahel Hirsch fellowship from the Charité Medical Faculty.

1 Bashiardes S, Veile R, Helms C, Mardis ER, Bowcock AM, Lovett M: Direct genomic selection. Nat Methods 2005; 2: 63-69.

2 Albert TJ, Molla MN, Muzny DM et al: Direct selection of human genomic loci by microarray hybridization. Nat Methods 2007; 4: 903-905.

3 Hodges E, Xuan Z, Balija V et al: Genome-wide in situ exon capture for selective resequencing. Nat Genet 2007; 39: 1522-1527.

4 Okou DT, Steinberg KM, Middle C, Cutler DJ, Albert TJ, Zwick ME: Microarraybased genomic selection for high-throughput resequencing. Nat Methods 2007; 4: 907-909.

5 Porreca GJ, Zhang K, Li JB et al: Multiplex amplification of large sets of human exons. Nat Methods 2007; 4: 931-936.

6 Herman DS, Hovingh GK, lartchouk 0 et al: Filter-based hybridization capture of subgenomes enables resequencing and copy-number detection. Nat Methods 2009; 6: 507-510.

7 Mardis ER: New strategies and emerging technologies for massively parallel sequencing: applications in medical research. Genome Med 2009; 1: 40.

8 Baird DM, Rowson J, Wynford-Thomas D, Kipling D: Extensive allelic variation and ultrashort telomeres in senescent human cells. Nat Genet 2003; 33: 203-207.

9 Hemann MT, Strong MA, Hao LY, Greider CW: The shortest telomere, not average telomere length, is critical for cell viability and chromosome stability. Cel/ 2001; 107: $67-77$.

10 Linardopoulou EV, Williams EM, Fan Y, Friedman C, Young JM, Trask BJ: Human subtelomeres are hot spots of interchromosomal recombination and segmental duplication. Nature 2005; 437: 94-100.

11 Lemmers RJ, Wohlgemuth M, van der Gaag KJ et al: Specific sequence variations within the $4 q 35$ region are associated with facioscapulohumeral muscular dystrophy. Am J Hum Genet 2007; 81: 884-894.

12 Hoskins RA, Carlson JW, Kennedy $\mathrm{C}$ et al: Sequence finishing and mapping of Drosophila melanogaster heterochromatin. Science 2007; 316: 1625-1628.

13 Dernburg AF, Broman KW, Fung JC et al: Perturbation of nuclear architecture by longdistance chromosome interactions. Cell 1996; 85: 745-759. 
14 Millington K, Hudnall SD, Northup J, Panova N, Velagaleti G: Role of chromosome 1 pericentric heterochromatin (1q) in pathogenesis of myelodysplastic syndromes: report of 2 new cases. Exp Mol Pathol 2008; 84: 189-193.

15 Talbert PB, Henikoff S: Spreading of silent chromatin: inaction at a distance. Nat Rev Genet 2006; 7: 793-803.

16 Helgadottir A, Thorleifsson G, Manolescu A et al: A common variant on chromosome 9p21 affects the risk of myocardial infarction. Science 2007; 316: 1491-1493.

17 Samani NJ, Erdmann J, Hall AS et al: Genomewide association analysis of coronary artery disease. N Engl J Med 2007; 357: 443-453.

18 Bahring S, Rauch A, Toka $O$ et al: Autosomal-dominant hypertension with type $E$ brachydactyly is caused by rearrangement on the short arm of chromosome 12 . Hypertension 2004; 43: 471-476.

19 Lunyak VV, Prefontaine GG, Nunez E et al: Developmentally regulated activation of a SINE B2 repeat as a domain boundary in organogenesis. Science 2007; 317: 248-251.

20 Varon R, Gooding R, Steglich C et al: Partial deficiency of the C-terminal-domain phosphatase of RNA polymerase II is associated with congenital cataracts facial dysmorphism neuropathy syndrome. Nat Genet 2003; 35: 185-189.

21 Mitelman F, Johansson B, Mertens F: The impact of translocations and gene fusions on cancer causation. Nat Rev Cancer 2007; 7: 233-245.

22 Tomlins SA, Laxman B, Dhanasekaran SM et al: Distinct classes of chromosomal rearrangements create oncogenic ETS gene fusions in prostate cancer. Nature 2007; 448: 595-599.

23 Campbell PJ, Stephens PJ, Pleasance ED et al: Identification of somatically acquired rearrangements in cancer using genome-wide massively parallel paired-end sequencing. Nat Genet 2008; 40: 722-729.
24 Weise A, Mrasek K, Fickelscher I et al: Molecular definition of high resolution multicolor banding (MCB) probes: first within the human dna-sequence anchored fish-banding probe set. J Histochem Cytochem 2008; 56: 487-493.

25 Telenius H, Carter NP, Bebb CE, Nordenskjold M, Ponder BA, Tunnacliffe A: Degenerate oligonucleotide-primed PCR: general amplification of target DNA by a single degenerate primer. Genomics 1992; 13: 718-725.

26 Liehr T, Weise A, Heller A et al: Multicolor chromosome banding (MCB) with YAC/BACbased probes and region-specific microdissection DNA libraries. Cytogenet Genome Res 2002; 97: 43-50.

27 Wheeler DA, Srinivasan M, Egholm M et al: The complete genome of an individual by massively parallel DNA sequencing. Nature 2008; 452: 872-876.

28 Harismendy O, Ng PC, Strausberg RL et al: Evaluation of next generation sequencing platforms for population targeted sequencing studies. Genome Biol 2009; 10: R32.

29 Sorek R, Zhu Y, Creevey CJ, Francino MP, Bork P, Rubin EM: Genome-wide experimental determination of barriers to horizontal gene transfer. Science 2007; 318: 1449-1452.

30 Amann J, Valentine M, Kidd VJ, Lahti JM: Localization of chi1-related helicase genes to human chromosome regions $12 \mathrm{p} 11$ and $12 \mathrm{p} 13$ : similarity between parts of these genes and conserved human telomeric-associated DNA. Genomics 1996; 32: 260-265.

31 Bailey JA, Gu Z, Clark RA et al: Recent segmental duplications in the human genome. Science 2002; 297: 1003-1007.

32 Lundin LG: Evolution of the vertebrate genome as reflected in paralogous chromosomal regions in man and the house mouse. Genomics 1993; 16: 1-19.

33 Zhang L, Lu HH, Chung WY, Yang J, Li WH: Patterns of segmental duplication in the human genome. Mol Biol Evol 2005; 22: 135-141.

Supplementary Information accompanies the paper on European Journal of Human Genetics website (http://www.nature.com/ejhg) 